\title{
Gravidez não planejada no extremo Sul do Brasil: prevalência e fatores associados
}

\author{
Unplanned pregnancy in Southern Brazil: \\ prevalence and associated factors
}

\author{
${ }^{1}$ Faculdade de Medicina, \\ Universidade Federal do Rio \\ Grande, Rio Grande, Brasil. \\ 2 Programa de Pós-graduação \\ em Nutrição, Universidade \\ Federal de Santa Catarina, \\ Florianópolis, Brasil. \\ Correspondência \\ S. O. M. Prietsch \\ Divisão de População \\ \& Saúde, Faculdade de \\ Medicina, Universidade \\ Federal do Rio Grande. \\ Av. Silva Paes 429 , \\ Rio Grande, RS \\ 96200-340, Brasil.
} silvio@brturbo.com.br

\section{Abstract}

The purpose of this study was to investigate factors associated with unplanned pregnancies in Rio Grande, Rio Grande do Sul State, Brazil. A standardized questionnaire was applied in 2007 to all pregnant women in the city, including demographic characteristics, childbearing history, socioeconomic status, and prenatal and childbirth care. The study used Poisson multivariate regression analysis with robust adjustment of variance. Among the 2,557 women included in the study, 65\% had not planned the current pregnancy. After adjusting for confounders, the following variables were significantly associated with unplanned pregnancy: black or mixed race, age $<20$ years, single marital status, low family income, household crowding, smoking, and multiparity. Previous abortion was a protective factor against unplanned pregnancy. The high unplanned pregnancy rate, especially among women with increased risk of complications during pregnancy and childbirth, highlights the need to target healthcare programs for this group.

Unplanned Pregnancy; Contraception; Adolescent; Maternal and Child Health
Silvio Omar Macedo Prietsch 1 David Alejandro González-Chica 2 Juraci A. Cesar 1 Raúl Andrés Mendoza-Sassi 1

\section{Introdução}

A cada ano, pelo menos 80 milhões de mulheres em todo o mundo experimentam a situação de ter uma gravidez não planejada, número que vem crescendo nas últimas décadas. A ocorrência desse fenômeno é responsável por um risco adicional no número de abortamentos e, além do episódio em si, aumenta o risco de morbidade e mortalidade ligadas ao aborto. Essa situação é bastante relevante na América do Sul, onde o número de procedimentos abortivos clandestinos está próximo dos quatro milhões por ano ${ }^{1}$.

Gravidez não planejada é toda a gestação que não foi programada pelo casal ou, pelo menos, pela mulher. Pode ser indesejada, quando se contrapõe aos desejos e às expectativas do casal, ou inoportuna, quando acontece em um momento considerado desfavorável. Ambas são responsáveis por uma série de agravos ligados à saúde reprodutiva materna e perinatal. A sua ocorrência tem impacto importante na oferta de cuidados de pré-natal, na orientação sobre aleitamento materno, no estado nutricional infantil e nas taxas de morbimortalidade materno-infantil. Embora pouco estudada, a gravidez não planejada representa risco aumentado de ansiedade e de depressão, sobretudo no período puerperal 2 .

A principal causa de gravidez indesejada, dentro de uma visão sociocultural laica, é o baixo índice de utilização de métodos contraceptivos. Este fator é mais frequente nos países pouco 
desenvolvidos, estando associado às dificuldades de acesso a serviços de saúde, à falta de organização destes ou a outros fenômenos sociais, como abuso sexual e coerção ${ }^{3}$. Dessa forma, a ocorrência de gravidez indesejada é uma questão relacionada ao direito fundamental da mulher sobre a sua fertilidade. O exercício desta prerrogativa não depende exclusivamente do acesso às informações ou aos métodos contraceptivos; passa pela possibilidade de tomar decisões em relação à sexualidade, à reprodução, como um aspecto da liberdade individual, influenciada diretamente por fatores socioeconômicos e culturais, e, em particular, à posição da mulher na sociedade 4,5 . Não existem evidências do verdadeiro papel dos fatores de risco individuais sobre o número de gestações indesejadas, principalmente porque são poucos os estudos a respeito dos ambientes sociais onde essas gestações ocorrem ${ }^{6}$.

Mesmo em países desenvolvidos, é elevado o índice de gravidez não planejada, embora haja diferenças metodológicas importantes no que se refere aos termos "indesejado" e "inoportuno". Estudo realizado nos Estados Unidos mostrou que $56 \%$ das gestações não são planejadas 7 .

Em uma análise de exposição de mulheres jovens à atividade sexual, foram demonstrados baixos índices de utilização de métodos contraceptivos. Essa pesquisa comparou a utilização desses métodos em oito países da América Latina e mostrou, no Brasil, um tempo "sexualmente ativo" protegido por anticoncepção de apenas $50 \%{ }^{8}$. É possível que o uso incorreto e inadequado de métodos anticoncepcionais responda pela maioria dos casos de insucesso na prevenção da gravidez, e não a falta de conhecimento sobre o método em si ${ }^{9}$. Estudo conduzido na Região Nordeste do Brasil sugere que os homens têm grande influência na escolha do método contraceptivo usado pelo casal, embora reconheçam que a responsabilidade da anticoncepção seja de ambos. Tal fato ainda não foi suficientemente explorado e se desconhece a verdadeira influência do pai no fenômeno da gravidez não planejada 10 .

Um grande estudo, recentemente conduzido em Gana, baseado em dados censitários, mostrou grande heterogeneidade geográfica entre comunidades rurais e urbanas e entre diversas regiões urbanas em relação ao risco de gravidez indesejada ou inoportuna. Esse resultado sugere que os fatores relacionados aos cenários sociais são importantes determinantes desta situação 11 .

Muitos outros fatores têm sido estudados para explicar esse aumento crítico no número de gestações indesejadas. Higgins et. al. 12 tentam explicar, por meio das teorias do comportamento, que sutis benefícios emocionais de características eróticas podem ser identificados, em al- guns casais, como negação de uso de métodos contraceptivos, ainda que subliminarmente. A transição para a parentalidade descrita por Pittman 13 pode ser considerada um fator altamente relevante. Segundo este autor, no passado os casais tinham maior consciência da iminência de serem pais, uma vez que a gravidez ocorria logo após o casamento e o início da vida sexual. Entretanto, em virtude do controle de natalidade e dos vínculos amorosos contemporâneos, em algumas localidades muitos casais não associam o início da atividade sexual com a parentalidade. Dessa maneira, paradoxalmente, o nascimento dos filhos, que poderia ser planejado com mais cuidado, e o planejamento consciente sobre o número e o momento de tê-los foram relegados a segundo plano.

O conceito de "planejamento familiar", instituído por Ponzetti Jr. ${ }^{14}$, inclui o momento do nascimento do primeiro filho, o intervalo de tempo entre os nascimentos e o momento de parar de ter filhos. Pode abarcar abortos, discussão dos diferentes tipos e significados de contracepção e testes, abrangendo, até mesmo, tratamentos de fertilidade. Tais cuidados estão, no Brasil, ao alcance apenas das famílias de melhor renda e/ ou que contam com um bom programa privado de saúde. O Programa de Assistência Integral à Saúde da Mulher (PAISM), iniciativa oficial do Ministério da Saúde, deveria ser entendido como uma política para assistência às mulheres no contexto do Sistema Único de Saúde (SUS), compreendendo toda a população feminina em idade fértil. Sua implementação resulta, em última instância, na qualificação dessa assistência e na melhoria dos níveis de saúde da população feminina. No entanto, o papel do Ministério da Saúde tem sido eminentemente normativo, cabendo às Secretarias Estaduais e Municipais de Saúde a execução dessas atividades, com autonomia para programar ou não as ações do PAISM. Logo, torna-se progressivamente necessária a disponibilidade de dados sobre o perfil socioeconômico, demográfico e reprodutivo das mulheres, tanto em nível nacional, como regional ou municipal, para um melhor planejamento dessas ações.

No Estado do Rio Grande do Sul, as ações do PAISM vêm sendo realizadas desde 1988. Atualmente, denominado como Sessão da Saúde da Mulher, prioriza suas ações no pré-natal, com especial atenção à gestante adolescente e à portadora do HIV, na assistência ao parto e ao puerpério, na prevenção de câncer do colo uterino e de mama e no planejamento familiar 15.

Para que as ações de planejamento familiar sejam desenvolvidas em nível local, é preciso o conhecimento das características das mulheres que apresentam gravidez indesejada, de forma 
a elaborar adequadas ações de saúde pública. Diante desse contexto, decidiu-se por avaliar o efeito de possíveis fatores associados à ocorrência de gravidez não planejada no Município de Rio Grande (Rio Grande do Sul). Para tanto, realizouse inquérito que incluiu todas as mulheres que tiveram partos entre os dias 1o de janeiro a 31 de dezembro de 2007 no município em questão.

\section{Metodologia}

Fizeram parte deste estudo todas as mães cujos partos ocorreram nas maternidades da Associação de Caridade Santa Casa do Rio Grande e do Hospital Universitário da Universidade Federal do Rio Grande (FURG), ao longo de 2007. Foram elegíveis para esta investigação todos os recémnascidos com peso ao nascer igual ou superior a $500 \mathrm{~g}$ ou 20 semanas ou mais de idade gestacional, cujas mães residissem no Município de Rio Grande. Foi utilizado um delineamento do tipo transversal, sendo as mães entrevistadas na maternidade por ocasião do parto.

Estimou-se que, dos cerca de 2.400 nascimentos ocorridos anualmente em Rio Grande, 99\% deles ocorreriam em uma dessas duas maternidades. Para incluir as crianças nascidas no domicílio, foi realizado rastreamento através do Sistema de Informações sobre Nascidos Vivos (SINASC).

Todos os dados foram coletados por meio de um único questionário pré-codificado, com a quase totalidade das perguntas fechadas. Esse questionário foi aplicado à mãe em até 24 horas após o parto, constando no instrumento dados sobre identificação, local de residência, características demográficas, ocupação, história reprodutiva e hábitos de vida dessas mulheres; nível socioeconômico, posse de eletrodomésticos, condições de habitação e saneamento de suas famílias; características do trabalho do pai; assistência recebida durante a gestação e o parto; acesso e utilização de serviços preventivos e curativos em saúde; conhecimento sobre amamentação e dieta, imunização e identificação de doença respiratória baixa.

Realizou-se, ainda, exame físico da criança, ocasião em que foram obtidas as seguintes medidas: comprimento, perímetro cefálico, perímetro torácico e circunferência abdominal. Para o comprimento, foi utilizado um infantômetro Harpenden (Holtain, Crymych, Reino Unido) com precisão de $1 \mathrm{~mm}$ e técnica padronizada; os perímetros foram medidos com fita métrica inelástica com precisão de $1 \mathrm{~mm}$, observando-se procedimento padronizado. $\mathrm{O}$ peso ao nascer foi copiado do livro de registro da enfermagem, a qual utilizou, nos dois hospitais, balança eletrônica pediátrica (Soehnle, London, Reino Unido) com precisão de $10 \mathrm{~g}$. As balanças foram aferidas semanalmente por integrantes do projeto, utilizando pesos-padrão. Além desta avaliação, baseada em características físicas e neurológicas do recém-nascido, a idade gestacional foi também calculada a partir da data da última menstruação (DUM) anotada no cartão da gestante, ou referida pela mãe (nesta ordem de prioridade), e por ultrassonografia realizada antes de vinte semanas de gestação.

Dez entrevistadores com, pelo menos, Ensino Médio completo foram treinados durante cinco dias consecutivos. $\mathrm{O}$ treinamento consistiu na leitura do questionário e do manual de instruções. Em seguida, foi realizado o estudo-piloto nas duas maternidades da cidade com o objetivo de testar não só o questionário a ser utilizado neste estudo, como também o enunciado de cada questão. Além disso, serviu para avaliar a logística do estudo.

Para o controle de qualidade, foram refeitas $5 \%$ das entrevistas com as mães nas residências ou ainda no hospital, utilizando-se um questionário resumido. A digitação dos questionários seguiu uma estratégia de entrada dupla, por dois digitadores independentes, utilizando o programa de computação Epi Info 6.4 (Centers for Disease Control and Prevention, Atlanta, Estados Unidos). Os dados foram verificados quanto à consistência, sendo acumulados em um banco para a criação de variáveis derivadas mediante o programa Stata 9 (Stata Corp., College Station, Estados Unidos). Um termo de consentimento foi obtido e assinado pela puérpera, autorizando a realização da entrevista e a coleta dos dados do recém-nascido. O protocolo de pesquisa foi devidamente aprovado pelo Comitê de Ética em Pesquisa na Área da Saúde (CEPAS) da FURG.

Para a análise dos dados, foi empregado o teste de Wald de heterogeneidade e de tendência para variáveis com mais de duas categorias e com orientação linear. Para a análise ajustada, foi utilizado um modelo hierarquizado através da regressão de Poisson com ajuste robusto da variância. Neste modelo, as variáveis são testadas de acordo com sua proximidade do desfecho, primeiro as mais distais e sucessivamente até as mais proximais à variável dependente (Figura 1). Foram mantidas no modelo de regressão todas as variáveis que apresentaram valor de $\mathrm{p} \leq 0,20$ na análise bruta. 


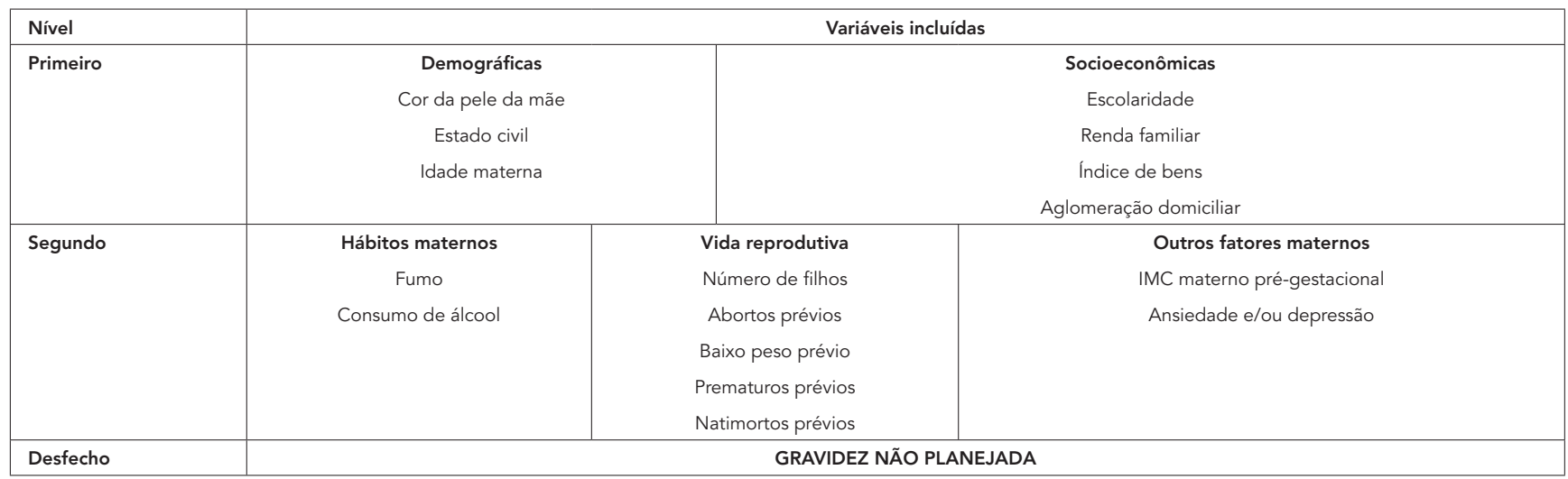

\section{Resultados}

Ao longo de 2007, foram entrevistadas 2.557 puérperas, de um total de 2.584, o que demonstra taxa de resposta da ordem de 99\%. Das entrevistadas, 2.023 (79\%) foram atendidas pelo SUS e as demais, por seguros de saúde privados ou particulares. De todas as gestações, $65 \%$ não foram planejadas.

A Tabela 1 mostra a análise bruta e ajustada para as variáveis socioeconômicas, demográficas e de moradia. Cor da pele preta/parda mostrou-se associada à gravidez não planejada. Para a cor da pele preta, a razão de prevalência (RP) foi de 1,23 (IC95\%: 1,14-1,32) na análise bruta e de 1,12 (IC95\%: 1,03-1,21) na análise ajustada. Dados semelhantes apareceram para aquelas de cor da pele parda, embora com menor RP. Quanto à idade da mãe, observou-se associação com a faixa etária que corresponde às adolescentes (13 a 19 anos), tanto na análise bivariada com RP de 1,23 (IC95\%: 1,14-1,34), quanto na análise ajustada (RP = 1,13; IC95\%: 1,04-1,23). Não ter companheiro apareceu associado à gravidez não planejada na análise bruta $(\mathrm{RP}=1,46$; IC95: 1,38 $1,54)$ e na análise ajustada $(\mathrm{RP}=1,29$; IC95: 1,22 1,37). Em relação aos fatores socioeconômicos, observou-se que as variáveis escolaridade materna, renda familiar e índice de bens apresentaram associação por meio do teste de tendência linear na análise bivariada com valor de $\mathrm{p}<0,001$. Na análise multivariada, essa tendência se manteve nas variáveis renda familiar $(\mathrm{p}=0,001)$ e índice de bens $(p<0,001)$. No que se refere ao número de moradores no domicílio, observou-se que quanto maior o número de moradores no domicílio, mais frequente foi a gravidez indesejada. A RP para três a quatro moradores no domicílio foi de 1,36 (IC95\%: 1,25-1,48) nas análises bruta e ajustada; para cinco ou mais moradores foi de 1,67 (IC95\%: 1,54-1,82) e de 1,57 (IC95\%: 1,45-1,72), respectivamente.

A Tabela 2 mostra a análise bruta e ajustada de acordo com hábitos maternos, antecedentes reprodutivos, presença de ansiedade e/ou depressão e índice de massa corporal (IMC) prégestacional. Mães que fizeram uso regular de tabaco tiveram mais gravidez indesejada, com RP de 1,21 (IC95\%: 1,14-1,28) na análise bivariada e de 1,08 (IC95\%: 1,02-1,15) na multivariada. Quanto ao consumo de álcool, apareceu uma associação na análise bruta, com RP de 1,16 (IC95\%: 1,03-1,16), que desapareceu completamente após o ajuste para as variáveis do primeiro nível. No que tange aos antecedentes reprodutivos, observou-se que as mulheres com mais filhos têm mais gravidez indesejada. Ter um ou dois filhos não apresentou associação na análise bruta, mas mostrou uma RP de 1,10 (IC95\%: 1,01$1,20)$ na análise ajustada. Para as mães com três ou mais filhos, a RP na análise bivariada é de 1,33 (IC95\%: 1,25-1,43) e de 1,44 (IC95\%: 1,30-1,59) na multivariada. Ter tido aborto previamente apareceu como associação negativa para gravidez não planejada, com RP de 0,90 (IC95\%: 0,83-0,98) na análise bruta e de 0,87 (IC95\%: 0,80-95) na análise ajustada. Ter tido partos que resultaram em fetos mortos não apresentou associação com 
Tabela 1

Análise bruta e ajustada da prevalência de gravidez não planejada conforme variáveis socioeconômicas, demográficas e de moradia. Rio Grande, Rio Grande do Sul, Brasil, 2007

\begin{tabular}{|c|c|c|c|c|}
\hline Variáveis & $\mathbf{n}$ & $\begin{array}{l}\text { Gravidez não } \\
\text { planejada (\%) }\end{array}$ & $\begin{array}{l}\text { Análise bruta } \\
\text { RP (IC95\%) }\end{array}$ & $\begin{array}{c}\text { Análise ajustada } \\
\text { RP (IC95\%) }\end{array}$ \\
\hline Cor da pele * & & & $p<0,001$ ** & $p=0,02$ ** \\
\hline Branca & 1.778 & 59,8 & 1,00 & 1,00 \\
\hline Parda/mulata & 468 & 63,4 & $1,14(1,06-1,23)$ & $1,05(0,98-1,13)$ \\
\hline Negra & 311 & 73,3 & $1,23(1,14-1,32)$ & $1,12(1,03-1,21)$ \\
\hline Idade (anos) * & & & $p<0,001 * \star$ & $p=0,006 * \star$ \\
\hline 13-19 & 516 & 72,7 & $1,23(1,14-1,34)$ & $1,13(1,04-1,23)$ \\
\hline $20-24$ & 718 & 64,5 & $1,09(1,01-1,19)$ & $1,09(1,00-1,18)$ \\
\hline $25-29$ & 628 & 57,8 & $0,98(0,89-1,07)$ & $1,00(0,92-1,09)$ \\
\hline$\geq 30$ & 695 & 59,0 & 1,00 & 1,00 \\
\hline Estado civil * & & & $p<0,001 * \star$ & $p<0,001 * \star$ \\
\hline Sem marido/Companheiro & 440 & 85,2 & $1,46(1,38-1,54)$ & $1,29(1,22-1,37)$ \\
\hline Com marido/Companheiro & 2.117 & 58,4 & 1,00 & 1,00 \\
\hline Escolaridade (anos) $\star \star \star$ & & & $p<0,001 \#$ & $p=0,5 \#$ \\
\hline $0-4$ & 322 & 67,4 & $1,29(1,12-1,49)$ & $0,95(0,82-1,10)$ \\
\hline $5-8$ & 923 & 67,5 & $1,29(1,14-1,47)$ & $0,97(0,84-1,11)$ \\
\hline $9-11$ & 1.071 & 60,2 & $1,15(1,01-1,31)$ & $0,97(0,85-1,10)$ \\
\hline 12 ou mais & 241 & 52,3 & 1,00 & 1,00 \\
\hline Renda familiar (Reais) ${ }^{\star \star \star}$ & & & $p<0,001 \#$ & $p=0,001 \#$ \\
\hline Tercil inferior & 858 & 69,5 & $1,23(1,14-1,32)$ & $1,13(1,05-1,21)$ \\
\hline Tercil médio & 847 & 62,8 & $1,11(1,02-1,20)$ & $1,08(1,00-1,16)$ \\
\hline Tercil superior & 852 & 56,7 & 1,00 & 1,00 \\
\hline Índice de bens * & & & $p<0,001 \#$ & $p<0,001 \#$ \\
\hline Tercil inferior & 852 & 69,0 & $1,25(1,16-1,35)$ & $1,15(1,07-1,24)$ \\
\hline Tercil médio & 852 & 64,8 & $1,18(1,09-1,27)$ & $1,11(1,03-1,20)$ \\
\hline Tercil superior & 851 & 55,1 & 1,00 & 1,00 \\
\hline Moradores no domicílio * & & & $p<0,001 \#$ & $p<0,001 \#$ \\
\hline $1-2$ & 754 & 47,4 & 1,00 & 1,00 \\
\hline $3-4$ & 1.171 & 64,3 & $1,36(1,25-1,48)$ & $1,36(1,25-1,48)$ \\
\hline 5 ou mais & 632 & 79,3 & $1,67(1,54-1,82)$ & $1,57(1,45-1,72)$ \\
\hline
\end{tabular}

* Variáveis conservadas no modelo quando o valor de $\mathrm{p}$ foi $\leq 0,20$ na análise ajustada;

** Teste de Wald de heterogeneidade;

*** Ajustado para outras variáveis do nível 1 com valor de $p \leq 0,20$, com exceção do índice de bens;

\# Teste de Wald de tendência.

gravidez não planejada, tanto na análise bruta quanto na análise ajustada. Da mesma forma, ter tido filhos prematuros ou com baixo peso ao nascer $(<2.500 \mathrm{~g})$ não mostraram associação na análise multivariada, embora a prematuridade tenha aparecido associada na análise bivariada com RP de 1,15 (IC95\%: 1,07-1,25). Antecedente de ansiedade e/ou depressão antes da gravidez não esteve associado à gravidez não planejada, tanto na análise bivariada, quanto na multivariada. O IMC pré-gestacional mostrou associação como fator protetor na análise bruta nas duas faixas mais altas de IMC $\left(25,0-29,9 \mathrm{~kg} / \mathrm{m}^{2}\right.$ $\mathrm{e} \geq 30,0 \mathrm{~kg} / \mathrm{m}^{2}$ ), com valor de $\mathrm{p}=0,002$. Este efeito não se manteve após o ajuste.

A Figura 2 mostra a reação do pai ao saber da gravidez e a Figura 3 mostra o apoio dado por ele à mãe durante a gestação. Estão significativamente associadas à gravidez não planejada a reação negativa do pai e a ausência de apoio deste durante a gestação $(\mathrm{p}<0,001)$.

O local onde as mães realizaram o pré-natal e o tipo de convênio disponível pela família estão significantemente associados com gravidez não planejada. A maioria das mães que não planejaram a gravidez foram as atendidas nos postos de 
Análise bruta e ajustada da prevalência de gravidez não planejada conforme hábitos maternos, antecedentes reprodutivos e índice de massa corporal (IMC) materno pré-gestacional. Rio Grande, Rio Grande do Sul, Brasil, 2007.

\begin{tabular}{|c|c|c|c|c|}
\hline Variáveis & $\mathrm{n}$ & $\begin{array}{l}\text { Gravidez não } \\
\text { planejada (\%) }\end{array}$ & $\begin{array}{l}\text { Análise bruta } \\
\text { RP (IC95\%) }\end{array}$ & $\begin{array}{c}\text { Análise ajustada *,** } \\
\text { RP (IC95\%) }\end{array}$ \\
\hline Fumo materno & & & $\mathrm{p}<0,001^{\star \star *}$ & $\mathrm{p}=0,008^{\star \star \star}$ \\
\hline Não & 1.846 & 59,5 & 1,00 & 1,00 \\
\hline $\operatorname{Sim}$ & 711 & 72,0 & $1,21(1,14-1,28)$ & $1,08(1,02-1,15)$ \\
\hline Consumo de álcool materno & & & $\mathrm{p}=0,02 \star \star \star$ & $p=1,0 \star \star \star$ \\
\hline Não & 2.461 & 62,6 & 1,00 & 1,00 \\
\hline $\operatorname{Sim}$ & 96 & 72,9 & $1,16(1,03-1,32)$ & $1,00(0,86-1,16)$ \\
\hline Número de filhos & & & $\mathrm{p}<0,001 * \star \star$ & $p<0,001 * \star \star *$ \\
\hline 0 & 1.010 & 57,9 & 1,00 & 1,00 \\
\hline $1-2$ & 874 & 57,9 & $1,00(0,93-1,08)$ & $1,10(1,01-1,20)$ \\
\hline$\geq 3$ & 673 & 77,3 & $1,33(1,25-1,43)$ & $1,44(1,30-1,59)$ \\
\hline Abortos prévios & & & $\mathrm{p}=0,01 \star \star \star$ & $p=0,001 * \star \star$ \\
\hline Não & 2.067 & 64,3 & 1,00 & 1,00 \\
\hline Sim & 490 & 57,8 & $0,90(0,83-0,98)$ & $0,87(0,80-0,95)$ \\
\hline Natimortos prévios & & & $\mathrm{p}=0,08 \star \star \star$ & $p=0,4 * \star \star$ \\
\hline Não & 2.468 & 62,7 & 1,00 & 1,00 \\
\hline Sim & 89 & 70,8 & $1,13(0,98-1,29)$ & $0,94(0,79-0,96)$ \\
\hline Prematuros prévios & & & $\mathrm{p}<0,001^{\star \star \star}$ & $\mathrm{p}=0,4 * \star \star$ \\
\hline Não & 2.259 & 61,9 & 1,00 & 1,00 \\
\hline Sim & 298 & 71,5 & $1,15(1,07-1,25)$ & $1,04(0,94-1,15)$ \\
\hline Baixo peso ao nascer prévio & & & $\mathrm{p}=0,3 \star \star \star$ & $\mathrm{p}=0,9 * \star \star$ \\
\hline Não & 2.264 & 62,1 & 1,00 & 1,00 \\
\hline Sim & 293 & 70,3 & $0,92(0,78-1,07)$ & $0,99(0,88-1,12)$ \\
\hline Ansiedade e/ou depressão (antes da gravidez) & & & $p=0,4 * \star \star$ & $p=0,6 * \star \star$ \\
\hline Não & 2.359 & 62,8 & 1,00 & 1,00 \\
\hline Sim & 180 & 66,1 & $1,05(0,94-1,17)$ & $0,96(0,84-1,10)$ \\
\hline IMC pré-gestacional (kg/m²) & & & $p=0,002 * \star \star$ & $p=0,2 \star \star \star$ \\
\hline$<18,5$ & 149 & 67,1 & 1,00 & 1,00 \\
\hline $18,5-24,9$ & 1.247 & 62,2 & $0,93(0,82-1,04)$ & $1,04(0,92-1,17)$ \\
\hline $25,0-29,9$ & 437 & 56,3 & $0,84(0,73-0,96)$ & $0,95(0,83-1,09)$ \\
\hline$\geq 30,0$ & 195 & 53,9 & $0,80(0,68-0,95)$ & $0,95(0,80-1,13)$ \\
\hline
\end{tabular}

* Ajustado para variáveis do primeiro nível: cor da pele, idade materna, índice de bens e número de moradores no domicílio;

** Variáveis conservadas no modelo quando o valor de $p$ foi $\leq 0,20$ na análise ajustada;

*** Teste de Wald de heterogeneidade.

saúde públicos e ambulatórios do SUS e tiveram seus filhos também pelo SUS $(\mathrm{p}<0,001)$.

\section{Discussão}

Foi a primeira vez que a prevalência de gravidez não planejada foi estimada em um estudo de base populacional no Município de Rio Grande. Essa informação é particularmente importante quando se necessita estabelecer programas de assistência à saúde materna e perinatal, uma vez que os ín- dices de morbidade e mortalidade nesta área são, ainda, elevados nos países em desenvolvimento. Ademais, em relação ao planejamento da gravidez, as mulheres com gravidez não desejada e seus filhos estão mais vulneráveis a diversos fatores de risco gestacionais e perinatais, especialmente entre os mais pobres 16 .

Este estudo não foi delineado para investigar diferentes aspectos de uma gravidez não planejada, não sendo capaz, em princípio, de diferenciar as gravidezes que foram indesejadas das que foram inoportunas. As noções de oportunidade, 


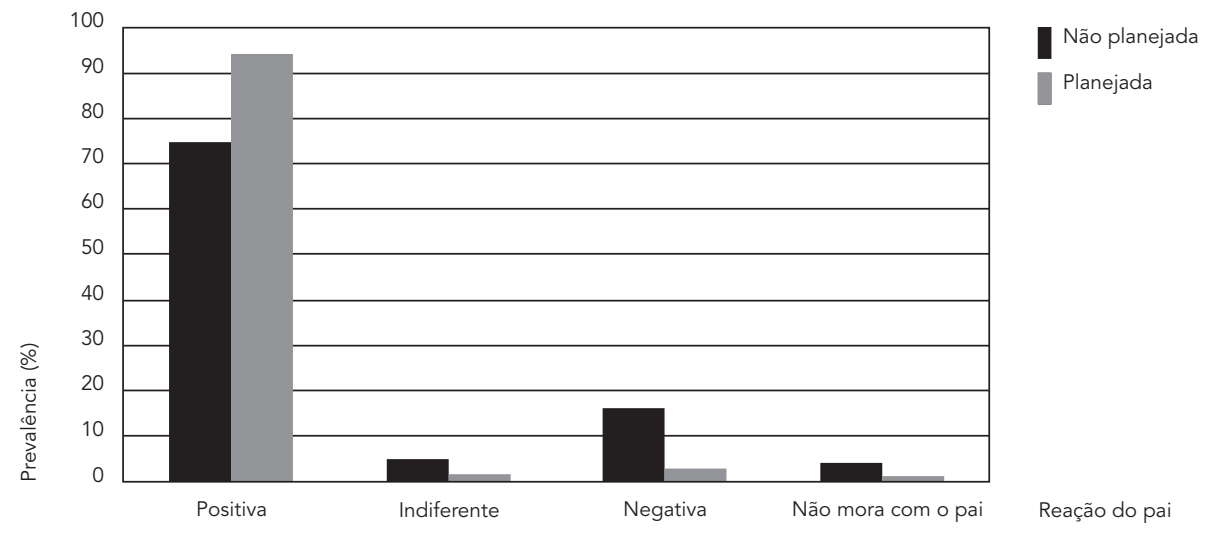

Nota: valor de $p<0,001$

Figura 3

Apoio do pai do bebê diante de uma gravidez planejada ou não planejada.

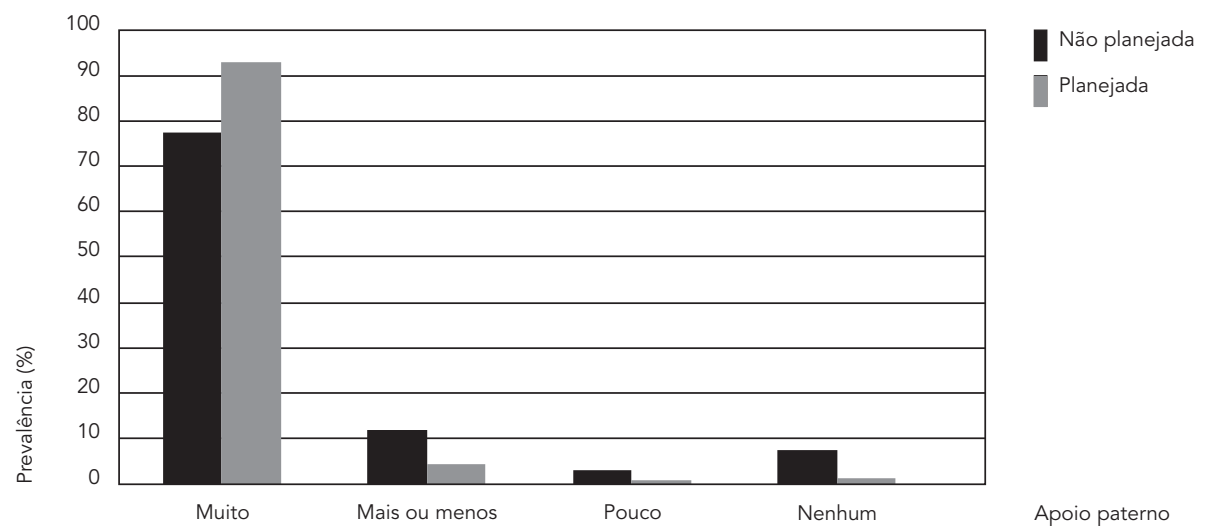

Nota: valor de $p<0,001$

adequação, previsão e desejabilidade desse evento não foram contempladas suficientemente de maneira a assegurar um diagnóstico cronobiológico de condição reprodutiva. No entanto, como os dados foram coletados no momento do nascimento, a diferenciação primária entre os dois tipos de gravidez não desejada está minimizada pela possibilidade de mudança dos sentimentos das mulheres, em relação aos seus conceptos, durante a gestação.
A maioria dos programas de intervenção, destinados a melhorar o acesso à contracepção, têm usado como indicadores de sucesso as taxas de fecundidade de um país ou região. Contudo, esses indicadores são menos informativos quando se quer avaliar o direito de decisão das mulheres de quando engravidar, cuja forma mais acurada é a medida da intenção da gravidez 6 .

No que se refere à gravidez não planejada, a prevalência de $65 \%$ encontrada no presente es- 
tudo é uma ocorrência bastante elevada, estando bem próxima da taxa descrita por Goicolea \& San Sebastian ${ }^{6}$, para o Equador (62,7\%), e por Gadow et al. 16, para a América do Sul (49,8\%). Ao se compararem esses resultados aos de países desenvolvidos, observa-se diferença importante. Dados coletados nos Estados Unidos mostram prevalências de gravidez acidental em torno de $60 \%$ quando os dados são coletados próximo do momento da concepção, mas essa taxa diminui para cerca de $30 \%$ quando coletados no momento do parto ${ }^{3}$. Em um estudo maior, que incluiu quatro estados americanos (Maine, Michigan, Oklahoma e Virginia Oeste), a prevalência de gravidezes inoportunas e indesejadas foi de $30 \% \mathrm{e}$ $9,7 \%$, respectivamente, atingindo o total de $39,7 \%$ de gravidezes não planejadas 17 .

O presente estudo foi também capaz de identificar alguns fatores associados à gravidez não planejada, demonstrando que esses eventos não são completamente aleatórios e estão, de alguma forma, ligados ao desejo de engravidar. As mulheres de cor da pele preta ou parda, as mais jovens, as que não vivem maritalmente, as mais pobres, as que vivem com mais de três pessoas em seus domicílios, as fumantes, as que já tinham filhos, as que não tiveram apoio dos pais de seus filhos e as não assistidas por planos de saúde privados foram as que apresentaram maior probabilidade de terem tido gravidez não planejada. $\mathrm{O}$ fato de ter tido um ou mais episódio de aborto prévio mostrou associação de proteção para gravidez não planejada.

Uma vez que esta pesquisa considerou todos os partos e recém-nascidos do município no ano de 2007, todas as situações geográficas, comunitárias e socioeconômicas foram representadas na mesma proporção do universo em estudo. A maioria dos fatores de risco estudados já havia sido descrita em estudos anteriores no Brasil 15 e em outros contextos 6 .

As duas variáveis que caracterizavam cor não branca (parda/mulata e preta) mostraram associação positiva com gravidez não planejada na análise bruta, mas esse efeito só permaneceu para as mulheres de cor negra após o ajuste para outros fatores demográficos e socioeconômicos na análise multivariada. Este é, provavelmente, um indicador de que as mulheres negras ainda sofrem algum tipo de desigualdade na realização plena de seus direitos reprodutivos, dependente não só da rede de serviços que lhes são oferecidos, mas, também, de determinantes sociais capazes de afetar a sua saúde reprodutiva 5,18 .

Foi observado que, quanto mais jovens são as mulheres, maior é a associação com gravidez não planejada. Esse efeito permanece após o ajuste para as outras variáveis demográficas e para as variáveis socioeconômicas nas mães com idades de 13 a 19 anos. A maioria dos estudos de gravidez não planejada mostra esse resultado $8,15,19$, embora um grande trabalho multicêntrico realizado na América do Sul tenha apontado, surpreendentemente, a idade mais avançada das mães como fator de risco para gravidez não planejada 16 . O conhecimento da maior prevalência de gravidez não planejada entre as adolescentes é um achado relevante, pois muitos programas para prevenção de gravidez não levam em consideração o risco de gravidez indesejada nessa faixa etária 6 .

Ser solteira ou não ter companheiro como fator de risco para gravidez não planejada não é um achado surpreendente, uma vez que a gravidez sem uma estruturação familiar não é uma condição patológica em si, mas uma problematização sociocultural ampla que merece mais atenção. Esse achado é semelhante ao encontrado em outros estudos 6,8,15,19 e pode ser um sinal de desigualdades entre os sexos, o que afeta as decisões das mulheres em relação à sua autonomia reprodutiva 10,18 .

Quanto aos fatores socioeconômicos estudados, foi possível observar que renda familiar e índice de bens apresentam efeito semelhante para gravidez não desejada, tendo associação positiva e podendo ser utilizados independentemente no modelo de análise multivariada. A maioria dos estudos identifica o baixo nível socioeconômico como fator de risco para gravidez não desejada, mesmo que esse efeito possa não ser demonstrado quando o universo de pesquisa é muito uniforme, como no caso de ser formado por uma parcela muito pobre da população, tal qual a descrita nos estudos realizados no Equador 6 e em Gana 20. A principal explicação para a associação positiva da baixa renda familiar com a gravidez não planejada parece estar relacionada com o uso incorreto ou a não utilização de métodos anticoncepcionais. Nem sempre tais métodos estão disponíveis e nem sempre há informação suficiente da mulher, seu parceiro, sua família ou comunidade para a escolha correta do método, o que leva à redução do tempo sexualmente ativo protegido por contraceptivo ${ }^{8}$.

No presente estudo, identificou-se, ainda, que já ter tido filhos aumenta o risco de gravidez não planejada. Esse efeito é maior, quanto maior o número de filhos tidos, apresentando-se independentemente associado na análise bivariada após o ajuste para as variáveis socioeconômicas, demográficas e de moradia. Esse achado está em conformidade com o descrito em outros estudos 6 , podendo ser um sinal de desconexão entre saúde materno-infantil e os serviços de planejamento familiar disponíveis.

A reação do pai e o apoio dado ao saber da gestação também se mostraram significativamente 
associados à gravidez não planejada neste estudo. Tal achado é o que se esperava dentro do contexto apresentado e está possivelmente relacionado com o comprometimento do pai com o recém-nascido. As mães cujos partos foram pelo SUS tiveram mais gravidezes não planejadas e são também as de menores condições socioeconômicas, possivelmente com maior dificuldade de acesso aos programas de planejamento familiar.

Este estudo detectou como potencial proteção o fato de ter ocorrido um ou mais episódios de abortamento prévios, efeito que permaneceu após o ajuste a todas as variáveis na análise multivariada. A maioria dos estudos trata o aborto como consequência da gravidez não planejada e não como fator determinante, uma vez que pode ser uma das principais consequências de gravidezes indesejadas 21,22 . Outros estudos excluem de suas análises a possibilidade de abortamento, já que, em grande parte dos países, em especial na América Latina, não há legalização do procedimento como método contraceptivo 2,6. Por outro lado, a inclusão da variável aborto, como no nosso estudo, mesmo sem especificar a possibilidade de ter sido um procedimento espontâneo ou induzido, pode produzir um viés importante, determinado pela própria ilegalidade e estigmatização social que a circunstância determina; por essa razão, esse achado deve ser visto com cautela.

Baixa escolaridade materna não mostrou associação significativa com gravidez não planejada após o ajuste para as demais variáveis socioeconômicas e variáveis demográficas. Goicolea \& San Sebastian ${ }^{6}$ encontraram uma associação significativa com duas vezes mais chance de gravidez indesejável entre as mulheres analfabetas, em comparação com as que tinham mais de nove anos completos de escolaridade. No estudo multicêntrico de Gadow et al. 16, ficou clara a associação de baixa escolaridade com gravidez indesejada; no entanto, para as gravidezes inoportunas, essa associação não pôde ser demonstrada.
Algumas limitações precisam ser consideradas ao interpretar os resultados do presente estudo. Uma delas é o corte transversal que caracteriza o desenho deste trabalho, o que dificulta a capacidade de identificar inferências causais entre os fatores estudados e o desfecho. Além disso, as informações coletadas foram autorreferidas por se tratar de questionamentos sobre desejo de ter tido seu filho, verificando-se uma espécie de relutância em classificá-los como indesejáveis ou inoportunos. Outro fator a considerar é que o sentimento no momento do parto pode ser completamente diferente daquele no momento do conhecimento da gravidez. Tal diferença provavelmente gera percentual menor do que o esperado se esse sentimento não tivesse mudado com o tempo.

Outra limitação importante é, sem dúvida, a impossibilidade de separar, no universo das gravidezes não planejadas, as indesejadas daquelas que foram simplesmente inoportunas. Kaufmann et al. 7 destacam que os determinantes de cada uma dessas situações podem ser distintos e ter consequências diversas no mecanismo psicossocial, tendo também importantes repercussões nas medidas intervencionistas para o planejamento familiar. Certamente, estudos complementares deverão ser realizados em Rio Grande para detalhar cada uma dessas situações, por meio da aplicação de instrumentos apropriados capazes de identificá-las.

Finalmente, a alta prevalência de gravidez não planejada em Rio Grande parece contradizer o fato estabelecido de que o uso crescente de métodos anticoncepcionais é uma intervenção eficaz para reduzir o número de gestações quando estas não deveriam acontecer. Este aspecto precisa ser considerado ao se avaliarem programas de planejamento familiar em funcionamento no município, sendo igualmente importantes informações sobre as taxas de conhecimento e de utilização de métodos contraceptivos pela população feminina sexualmente ativa. 


\section{Resumo}

Este estudo teve por objetivo identificar fatores associados à ocorrência de gravidez não planejada em Rio Grande, Rio Grande do Sul, Brasil. Em 2007, aplicouse questionário padronizado a todas as parturientes residentes neste município, investigando sobre características demográficas e reprodutivas maternas, nível socioeconômico da família e assistência recebida na gestação e parto. A análise ajustada foi realizada utilizando-se regressão de Poisson com ajuste robusto da variância. Dentre as 2.557 gestantes incluídas no estudo, 65\% não planejaram a gravidez. Após ajuste para confundimento, as seguintes variáveis mostraram-se significativamente associadas à gravidez não planejada: cor da pele parda/preta, idade inferior a 20 anos, sem companheiro, baixa renda familiar, aglomeração familiar, tabagismo e mais de um parto. Abortamento prévio mostrou-se protetor para a gravidez não planejada. A elevada ocorrência de gravidez não planejada, sobretudo entre aquelas com maiores riscos de complicações durante a gestação e o parto, indica a necessidade de se estabelecerem programas de saúde à atenção desta população.

Gravidez Não Planejada; Anticoncepção; Adolescente; Saúde Materno-Infantil

\section{Colaboradores}

S. O. M. Prietsch participou da concepção e projeto do artigo, análise dos dados, redação e aprovação da versão final. D. A. González-Chica colaborou na análise estatística e redação final do artigo. J. A. Cesar contribuiu na concepção e projeto do artigo, interpretação dos dados, revisão crítica do conteúdo, ajuda na aprovação final. R. A. Mendoza Sassi participou no projeto, análise dos dados, revisão do conteúdo e aprovação da versão final.

\section{Referências}

1. Langer A. El embarazo no deseado: impacto sobre la salud y la sociedad en América Latina y el Caribe. Rev Panam Salud Pública 2002; 11:192-203.

2. Gipson JD, Koenig MA, Hindin MJ. The effects of unintended pregnancy on infant, child, and parental health: a review of the literature. Stud Fam Plann 2008; 39:18-38.

3. Cleland J, Bernstein S, Ezeh A, Faundes A, Glasier A, Innis J. Family planning: the unfinished agenda. Lancet 2006; 368:1810-27.
4. Glasier A, Gülmezoglu AM, Schmid GP, Moreno CG, Van Look PF. Sexual and reproductive health: a matter of life and death. Lancet 2006; 368:1595-607.

5. Gruskin S. Reproductive and sexual rights: do words matter? Am J Public Health 2008; 98:1737.

6. Goicolea I, San Sebastian M. Unintended pregnancy in the amazon basin of Ecuador: a multilevel analysis. Int J Equity Health 2010; 9:14.

7. Kaufmann RB, Morris L, Spitz AM. Comparison of two question sequences for assessing pregnancy intentions. Am J Epidemiol 1997; 145:810-6. 
8. Ali M, Cleland J. Sexual and reproductive behaviour among single women aged 15-24 in eight Latin American countries: a comparative analysis. Soc Sci Med 2005; 60:1175-85.

9. Paniz VMV, Fassa ACG, Silva MC. Conhecimento sobre anticoncepcionais em uma população de 15 anos ou mais de uma cidade do Sul do Brasil. Cad Saúde Pública 2005; 21:1747-60.

10. Espírito-Santo DC, Tavares-Neto J. A visão masculina sobre métodos contraceptivos em uma comunidade rural da Bahia, Brasil. Cad Saúde Pública 2004; 20:562-9.

11. Amoako Johnson F, Madise N. Examining the geographical heterogeneity associated with risk of mistimed and unwanted pregnancy in Ghana. J Biosoc Sci 2009; 41:249-67.

12. Higgins JA, Hirsch JS, Trussell J. Pleasure, prophylaxis and procreation: a qualitative analysis of intermittent contraceptive use and unintended pregnancy. Perspect Sex Reprod Health 2008; 40:130-7.

13. Pittman F. Mentiras privadas. 3a Ed. Porto Alegre: Editora Artes Médicas; 1994.

14. Ponzetti Jr. J. Family planning. In: Ponzetti Jr. J, editor. International encyclopedia of marriage and family. New York: Thomson Gale; 2003. p. 612-5.

15. Olinto MT, Galvão LW. Características reprodutivas de mulheres de 15 a 49 anos: estudos comparativos e planejamento de ações. Rev Saúde Pública 1999; 33:64-72.
16. Gadow EC, Paz JE, López-Camelo JS, Dutra MG, Queenan JT, Simpson JL, et al. Unintended pregnancies in women delivering at 18 South American hospitals. NFP-ECLAMC Group. Latin American Collaborative Study of Congenital Malformations. Hum Reprod 1998; 13:1991-5.

17. Adams MM, Bruce FC, Shulman HB, Kendrick JS, Brogan DJ. Pregnancy planning and pre-conception counseling. The PRAMS Working Group. Obstet Gynecol 1993; 82:955-9.

18. Shaw D. Sexual and reproductive health: rights and responsibilities. Lancet 2006; 368:1941-3.

19. Belo MAV, Silva JLP. Conhecimento, atitude e prática sobre métodos anticoncepcionais entre adolescentes gestantes. Rev Saúde Pública 2004; 38:479-87.

20. Geelhoed D, Nayembil D, Asare K, van Leeuwen $\mathrm{JH}$, van Roosmalen J. Gender and unwanted pregnancy: a community-based study in rural Ghana. J Psychosom Obstet Gynaecol 2002; 23:249-55.

21. Fonseca W, Misago C, Correia LL, Parente JAM, Oliveira FC. Determinantes do aborto provocado entre mulheres admitidas em hospitais em localidade da região Nordeste do Brasil. Rev Saúde Pública 1996; 30:13-8.

22. Kuroki LM, Allsworth JE, Redding CA, Blume JD, Peipert JF. Is a previous unplanned pregnancy a risk factor for a subsequent unplanned pregnancy? Am J Obstet Gynecol 2008; 199:517.e1-7.

Recebido em 12/Nov/2010

Versão final reapresentada em 24/Mai/2011

Aprovado em 07/Jun/2011 